\title{
Hydrogen Bonding in a Reversible Comb Polymer Architecture: A Microscopic and Macroscopic Investigation
}

Mariapaola Staropoli ${ }^{1}$, Andreas Raba ${ }^{1}$, Claas H. Hövelmann ${ }^{1}$, Margarita Krutyeva ${ }^{1}$, Jürgen Allgaier ${ }^{1}$, Marie-Sousai Appavou ${ }^{2}$, Uwe Keiderling ${ }^{3}$, Florian J. Stadler ${ }^{4}$, Wim Pyckhout-Hintzen ${ }^{1}$, Andreas Wischnewski ${ }^{1}$, Dieter Richter ${ }^{1}$

1 JCNS-1 and ICS-1, Forschungszentrum Jülich GmbH, Leo-Brandt-Straße, 52425 Jülich, Germany

2 Jülich Centre for Neutron Science (JCNS) at MLZ, 85747 Garching, Germany

${ }^{3}$ Helmholtz-Zentrum Berlin für Materialien and Energie, 14109 Berlin, Germany

${ }^{4}$ Nanshan District Key Lab for Biopolymers and Safety Evaluation, College of Materials Science and Engineering and Department of Optoelectronic Engineering, Shenzhen University, Shenzhen, Guangdong, China

Abstract: In this work an investigation of the hydrogen bonding mechanism in a transiently branched comb-like polymer system in the melt is reported. The system under investigation consists of a polybutylene oxide (PBO)-based backbone, randomly functionalized with thymine (thy) groups, in combination with shorter PBO graft chains, end-functionalized with diaminotriazine (DAT) groups. The functional groups are able to associate through hydrogen bonding. The hetero-complementary association of these groups leads to the formation of a transiently branched comb-like polymer system. Since recently virtually exclusive heterocomplementary association could be observed in the supramolecular association of telechelically-modified oligomeric PEG chains, here we aim to extend the supramolecular assembly mechanism towards branched structures. The present work combines Small Angle Neutron Scattering (SANS) experiments on a selectively labeled system with macroscopic dynamics measured in linear rheology response. The association of thy- and DAT-modified components was studied as a function of temperature and composition. The scattering function reveals the formation of a block copolymer and can be exclusively attributed to heterocomplementary association of the hydrogen bonding groups. Scattering functions of nonfunctionalized blends are also reported as references and evidence the change in the microstructure induced by the hetero-complementary association. All scattering profiles were described by means of the Random Phase Approximation (RPA) formalism from which the average aggregation number i.e. comb arm functionality and the equilibrium association constant could be determined directly in the melt state as a function of temperature. On the other hand, rheological measurements were performed in the melt state to study the influence of the reversible bonds on the macroscopic dynamics of the polymer system. The rheology data are in good agreement with the SANS results and confirm the transient comb-like branched architecture. The supramolecular association exhibits characteristic bonding times of the groups in the order of $1 \mathrm{~s}$ at $-25^{\circ} \mathrm{C}$ and therefore makes the thy-DAT pair an ideal candidate for 
the development of responsive materials that combine permanent and transient linkages for novel applications and self-healing properties.

\section{Introduction:}

Supramolecular polymers, formed through weak secondary interactions between polymer chains, have gained an increasing interest in the last decades. ${ }^{1}$ Due to the reversibility of the secondary interactions, the topology as well as the mechanical properties of the polymer system can be controlled through external variables such as temperature or mechanical shear. The presence of weak interactions coexisting with covalent bonding can impart new interesting properties like e.g. self-healing, ${ }^{2}$ which allows the polymer system to form transient networks able to release local overstresses through disconnection of the transient bonds. After the programmed damage, they spontaneously re-associate and repair the structure. Due to the associated high directionality and reversibility the hydrogen bonding mechanism is the most prominently applied secondary interaction used in supramolecular chemistry. ${ }^{3}$ One of the simplest examples of associating polymers is expected with linear oligomeric polymer chains carrying end-functionalized binding groups which are able to form long supramolecular linear chains. This linear aggregation can be well described in terms of Flory's mean-field theory of polycondensation of classical monomers. While the association of building blocks bearing hydrogen bonding groups to form supramolecular architectures has been well studied in solution, ${ }^{4}$ much less is known for polymer melts. Recently, the association of bifunctional linear oligomeric chains bearing thymine (thy) and diaminotriazine (DAT) groups was studied both in solution and melt state. ${ }^{5,6}$ In solution, the hetero-complementary association of thy-DAT was shown to be dominant compared to the weaker (thy-thy, DAT-DAT) self-association. ${ }^{5}$ In the melt state, other mechanisms like phase segregation of the components or crystallization of

hydrogen bonding groups can compete with the supramolecular association. ${ }^{6}$ This is especially true in the case of a strongly different polarity of the polymer matrix and the stickers. Crystallization of functional groups and formation of unspecific clusters in the melt state were observed in PIB, a very nonpolar polymer matrix. ${ }^{6 a}$ However, only recently virtually exclusive hetero-complementary association could be observed in the supramolecular association of telechelically-modified PEG. ${ }^{7}$ Therein, the interaction parameters between the groups and the matrix were such that polarity effects could be minimized and only the hydrogen bonding was emphasized.

In the present work we aim to extend the linear supramolecular assembly towards the formation of branched structures, combining the supramolecular association property with properties that are typical for branched polymers. The system under investigation consists of a 
poly(1,2-butylene oxide) (PBO)-based linear chain randomly side-functionalized with thy groups in combination with PBO short graft chains bearing mono DAT end-functionalization. The medium polarity of the polymer matrix which is somewhat lower than that of PEG and the dilute presence of stickers along a long backbone is expected to decisively prevent clustering or micellization of the stickers. The hetero-complementary association of the hydrogen bonding groups would ideally lead to the formation of a transient comb structure which exhibits some characteristics of a covalently branched polymer. The Small Angle Neutron Scattering (SANS) technique was shown to be a unique method that is able to detect the microscopic structural aggregation of the groups into a temporary and dynamically-branched architecture. By means of a special isotopic labeling scheme (Fig.1) the dominant hetero-complementary association leads to the formation of a branched diblock copolymer for which a correlation peak is expected in the scattering function at intermediate q-range. This differs entirely from the scattering pattern of a random mixture of deuterated and hydrogenous inactive components, which would show a Debye-like behavior. The equilibrium between the associated components and the blend of linear chains depends on the strength of the hydrogen bonds and their arrangement and is furthermore affected by temperature. In the following, we develop and apply a quantitative Random Phase Approximation (RPA) analysis for a transient H,D - diblock copolymer to SANS data, obtained at different temperatures and different relative proportions of graft arms to backbone. The scattering data reveal an exclusive hetero-complementary association. The position and the intensity of the pronounced correlation peak are related to the size of the repeating $\mathrm{HDH}$ block and the average aggregation number as well as an isotopic Flory-Huggins interaction parameter due to the H-D labeling (Fig.1) with some additional enthalpic contribution due to the comb-like, non-linear association. ${ }^{8}$ To investigate the interaction parameters separate SANS experiments on non-functionalized blends were also conducted. In addition, the dynamics of the supramolecular comb and inactive components was investigated in linear rheology. Some self - association of the arms could be shown at low temperatures only. We were able to link the microscopic structure with the macroscopic linear response and detect a time scale of the order of about $1 \mathrm{~s}$ at $-25^{\circ} \mathrm{C}$ in which hydrogen bonds can associate and dissociate leading to a switch in the polymeric architecture from linear to branched and vice versa. The thy-DAT pair can therefore be considered as an interesting candidate for the development of new elastomers with built-in stiffener or plasticizer depending on the rate of the mechanical activation. 


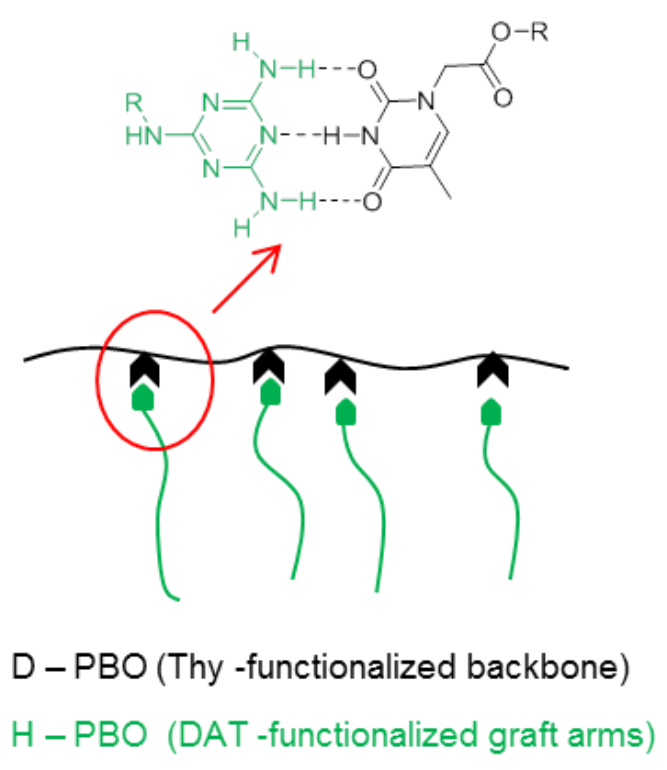

Figure 1: Scheme of the blend components and thy-DAT hydrogen-bonding interactions.

\section{Experimental Section}

2.1. Synthesis: The backbone sample $\mathbf{d}$-PBO $80 \mathbf{k}$ was synthesized by first randomly copolymerizing deuterated 1,2-butylene oxide and hydrogenous 1,2-epoxy-7-octene and described in the previous work. ${ }^{9}$ The polymerization reaction was terminated with methyl iodide in order to obtain polymer chains, free of terminal $\mathrm{OH}$-groups. The synthesis of the deuterated 1,2-butylene oxide monomer is described in literature. ${ }^{10} 6.84 \mathrm{~g}$ of the obtained copolymer $\left(\mathrm{M}_{\mathrm{n}}(\mathrm{SEC} / \mathrm{LS})=76,900 \mathrm{~g} / \mathrm{mol}, \mathrm{M}_{\mathrm{w}} / \mathrm{M}_{\mathrm{n}}(\mathrm{GPC})=1.02,1.50 \mathrm{mmol}\right.$ of vinyl groups) were dissolved in $40 \mathrm{~mL}$ of THF and 9-BBN solution $(10 \mathrm{~mL}, 4.60 \mathrm{mmol})$ was slowly added. The mixture was stirred for $16 \mathrm{~h}$ at $\mathrm{rt}$ and subsequently cooled to $0{ }^{\circ} \mathrm{C}$. Methanol $(4.0 \mathrm{~mL}$, $98.6 \mathrm{mmol}$ ) was added outside the glovebox and the mixture was stirred for $15 \mathrm{~min}$. Aqueous sodium hydroxide solution ( $6 \mathrm{~mol} / \mathrm{L} ; 6 \mathrm{~mL}, 36.0 \mathrm{mmol}$ ) was added, followed by hydrogen peroxide $(5 \mathrm{~mL}, 49 \mathrm{mmol})$ and the solution was allowed to stir at $\mathrm{rt}$ for $16 \mathrm{~h}$. After concentrating the solution, deionized water $(100 \mathrm{~mL})$ was added. The polymer phase was separated and washed three times with methanol to yield $6.22 \mathrm{~g}\left(91 \%, \mathrm{M}_{\mathrm{w}} / \mathrm{M}_{\mathrm{n}}(\mathrm{SEC})=1.03\right)$ of d-PBO 80k. The thymine- functionalized material $\mathbf{d}$-PBO thy-80k was synthesized as reported previously ${ }^{9}$ using d-PBO $80 \mathrm{k}$ as precursor $(11.63 \mathrm{~g}, 2.53 \mathrm{mmol}$ OH-groups), TBTU $(1.824 \mathrm{~g}, 5.68 \mathrm{mmol}), \mathrm{N}, \mathrm{N}-$ diisopropylethylamine $(2.34 \mathrm{~g}, 18.1 \mathrm{mmol})$ and thymine-1-acetic acid $(0.942 \mathrm{~g}, 5.12 \mathrm{mmol})$ as well as $25.5 \mathrm{~g}$ of dry THF and $51.5 \mathrm{~g}$ of dry DMF. 
The mixture was left for $2 \mathrm{~d}$ at rt. Yield: $7.1 \mathrm{~g}\left(61 \% ; \mathrm{M}_{\mathrm{w}} / \mathrm{M}_{n}(\mathrm{SEC})=1.10\right)$. The amount of thymine groups per chain was determined by comparison of the ${ }^{1} \mathrm{H}-\mathrm{NMR}$ Signals of the t-butyl group of the initiator and two thymine signals at 4.1 and $4.5 \mathrm{ppm}$ in $\mathrm{CDCl}_{3}$ to be 15.45 groups/chain. Analogous, a residual $\mathrm{OH}$ content of 2.33 groups/chain and 0.3 residual vinyl groups/chain were found by ${ }^{1} \mathrm{H}-\mathrm{NMR}$ in pyridine- $\mathrm{d}_{5}$ (thymine content: 15.45 groups/chain, $0.201 \mathrm{mmol} / \mathrm{g}$; degree of thymine functionalization: $86 \%,-\mathrm{OH}$ functionalization: $13 \%$, vinyl functionalization: $1 \%$, coupling product of double molecular weight: $8 \mathrm{wt} \%)$. This leads to a total functionalization of 18.05 groups/chain compared to a vinyl content of 18.2 groups/chain in the parent polymer.

The graft polymers PBO 5k and PBO 15k were synthesized following the procedure reported in literature. ${ }^{11}$ PBO 5k was obtained from 1,2-butylene oxide ( $\left.82.0 \mathrm{~g}\right), \mathrm{KOt}$-Bu (1.84 g, $\left.16.4 \mathrm{mmol}\right)$, $18 \mathrm{C} 6(2.14 \mathrm{~g}, 8.08 \mathrm{mmol})$ and dry toluene $(78 \mathrm{~g})$ polymerizing at a temperature of $-15^{\circ} \mathrm{C}$ for $3 \mathrm{~d}$. Isolated yield: $78.1 \mathrm{~g}$ (94\%; $\left.M_{n}(N M R)=5,080 \mathrm{~g} / \mathrm{mol}, M_{w} / M_{n}(S E C)=1.02\right)$. PBO 5k-DAT was synthesized starting from PBO5k- $\mathbf{N H}_{2}{ }^{9}\left(6.83 \mathrm{~g}, 1.35 \mathrm{mmol} ; \mathrm{M}_{\mathrm{n}}(\mathrm{NMR})=5,080 \mathrm{~g} / \mathrm{mol}\right.$, degree of $\mathrm{NH}_{2}$ functionalization: 96\%), DAT-Cl (364 mg, $\left.2.50 \mathrm{mmol}\right), \mathrm{NaHCO}_{3}(306 \mathrm{mg}, 3.64 \mathrm{mmol}$ ) and DMA (25 mL). Yield: $4.84 \mathrm{~g}\left(71 \%, \mathrm{M}_{\mathrm{w}} / \mathrm{M}_{\mathrm{n}}(\mathrm{SEC})=1.03\right.$, degree of DAT functionalization: $94 \%,-\mathrm{OH}$ functionalization: $2 \%,-\mathrm{NH}_{2}$ functionalization: $4 \%$, coupling product of double molecular weight: $5 \mathrm{wt} \%$ ) as a colorless liquid. PBO 15k was synthesized starting from 1,2-butylene oxide (33.3 g), $\mathrm{KOt}$-Bu (249 mg, $2.22 \mathrm{mmol}), 18 \mathrm{C} 6(587 \mathrm{mg}, 2.22 \mathrm{mmol})$ and dry toluene (33.7 g) and carrying out the polymerization at $-10{ }^{\circ} \mathrm{C}$ for $7 \mathrm{~d}$. Isolated yield: $32.8 \mathrm{~g}\left(98 \% ; M_{n}(N M R)=15,800 \mathrm{~g} / \mathrm{mol}\right.$, $\left.M_{w} / M_{n}(S E C)=1.01\right)$. This polymer was used as non-functionalized reference material. For the DAT-functionalized (PBO 15k-DAT) polymer another batch was synthesized using 1,2-butylene oxide (52.8 g), KOt-Bu (371 mg, $3.31 \mathrm{mmol}), 18 \mathrm{C} 6(865 \mathrm{mg}, 3.27 \mathrm{mmol}$ ) and dry toluene (59 g) and carrying out the polymerization at $-14^{\circ} \mathrm{C}$ for 2 days and at $-10^{\circ} \mathrm{C}$ for one day. $\left(\mathrm{M}_{\mathrm{n}}(\mathrm{NMR})=\right.$ $\left.14,100 \mathrm{~g} / \mathrm{mol}, \mathrm{M}_{\mathrm{w}} / \mathrm{M}_{\mathrm{n}}(\mathrm{SEC})=1.02\right)$. PBO15k-DAT was synthesized starting from $\mathbf{P B O} 15 \mathrm{k}-\mathbf{N H}_{\mathbf{2}}{ }^{9}$ (3.12 g, $\left.0.22 \mathrm{mmol} ; \mathrm{M}_{\mathrm{n}}(\mathrm{NMR})=14,100 \mathrm{~g} / \mathrm{mol}\right), \mathrm{DAT}-\mathrm{Cl}(64.4 \mathrm{mg}, 0.44 \mathrm{mmol}), \mathrm{NaHCO}_{3}(56 \mathrm{mg}$, $0.67 \mathrm{mmol})$ and DMA $(5 \mathrm{ml})$. Yield: $61 \%\left(\mathrm{M}_{\mathrm{w}} / \mathrm{M}_{\mathrm{n}}(\mathrm{SEC})=1.03\right.$, degree of $-\mathrm{DAT}$ functionalization: $89 \%,-\mathrm{OH}$ functionalization $6.5 \%,-\mathrm{NH}_{2}$ functionalization: $4.5 \%$, coupling product of double molecular weight: $4.5 \mathrm{wt} \%)$ 


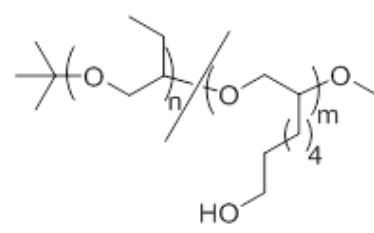

d-PBO 80k

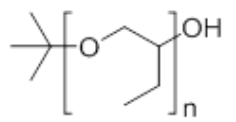

PBO 15k

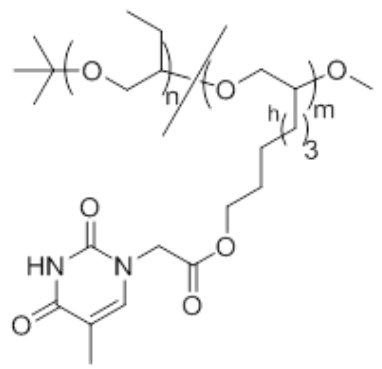

d-PBO thy $80 k$

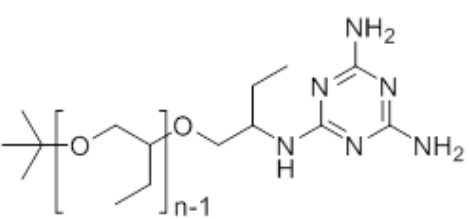

PBO 15k-DAT

Figure 2: Structure of PBO precursors and functionalized samples.

2.2. Sample Preparation: Three mixtures with different stoichiometric ratios of thy-DATmodified PBO were prepared. While the thymine-functionalized backbone is the deuterated component of the mixture, the arms are hydrogenous. The samples were prepared by mixing stock solutions in pentane. The solvent was removed under high vacuum for 4 days. As reference blends of non-functionalized components at the same stoichiometric ratios were prepared using the same procedure.

2.3. Small Angle Neutron Scattering: SANS experiments were performed using the KWS-2 diffractometer at MLZ (Garching, Germany) ${ }^{12}$ and the V4 diffractometer (HZB Berlin, Germany) using sample-to-detector distances between 20 and $1.35 \mathrm{~m}$ and $\lambda=5 \AA$, covering a q-range between $1 \cdot 10^{-1}$ and $3 \cdot 10^{-3} \AA^{-1}$ with $q=\frac{4 \pi}{\lambda} \sin \frac{\theta}{2}$ where $\theta$ is the scattering angle. For the absolute scaling to $\mathrm{cm}^{-1}$, the incoherent scattering level of Plexiglas ${ }^{\circledR}$ and water was used respectively. The two-dimensional data were corrected for empty cell scattering, detector sensitivity and background noise using $\mathrm{B}_{4} \mathrm{C}$ and $\mathrm{Cd}$ as beam blocker and radially averaged. The incoherent background was determined from the high q-range and was in good agreement with 
the estimated incoherent scattering of the hydrogenous polymer fraction. Because of the very small concentration of functional groups the contrast factor was calculated by smearing the scattering length densities of the polymer chains and the functional groups.

2.4. Rheology: Oscillatory shear experiments were carried out on a strain-controlled ARES (Rheometrics Sci Ltd) rheometer using the parallel plate geometry. The diameter of the plates was $8 \mathrm{~mm}$ or $25 \mathrm{~mm}$ and the sample gap about $1 \mathrm{~mm}$. Isothermal frequency sweeps were performed in the range between $0.1<\omega<100 \mathrm{rad} / \mathrm{s}$. A temperature range between $-55<\mathrm{T}<-5$ ${ }^{\circ} \mathrm{C}$ with $\Delta \mathrm{T}=10^{\circ} \mathrm{C}$ under a nitrogen blanket within the linear rheology regime of the polymers was chosen. Master curves were obtained at a reference temperature of $-25^{\circ} \mathrm{C}$ for each sample. In the case of the functionalized mixtures, the shift factor $\mathrm{a}_{\mathrm{T}}$ for the construction of the master curves follows to a very good approximation a WLF behavior indicating a rather simple thermo-rheological behavior. $T_{\mathrm{g}}$ values of all polymers were determined by DSC (Q2000, TA Instruments) at heating and cooling rates of $10^{\circ} \mathrm{C} / \mathrm{min}$ and differed only within $\Delta T_{g} \sim 2{ }^{\circ} \mathrm{C}$ : $T_{g}=(-65 \pm 2)^{\circ} \mathrm{C}$.

\section{Scattering model and description}

The scattering function of a supramolecular diblock copolymer resulting from the heterocomplementary association of the hydrogen bonding groups, which themselves are attached telechelically to hydrogenous and deuterated sequences is described by means of the Random Phase Approximation (RPA) formalism. The RPA applies in general to a binary blend of polymers with different scattering length density. The interactions between the components of the blend are quantified by a Flory - Huggins parameter $\chi$. Recently it was shown that it yields most sensitive structural information about the structure of supramolecular chains on the level of the constituting units. For simplicity our system will be considered as a two-component blend with a simple non-zero interaction parameter between the two components. The active end-groups are integrated into the polymer chain by extending it over a comparable monomer distance and averaging over the scattering length, in the same way as in reference [7]. Following literature the RPA expression for a diblock copolymer is written in terms of the bare non-interacting structure factors where we dropped the q-dependence for clarity only as follows: ${ }^{13}$

$\frac{d \Sigma}{d \Omega} / \frac{\Delta \rho^{2}}{N_{A}}=S_{R P A}=\left(S_{i i} \cdot S_{a a}-\left(S_{i a}^{2}\right)\right) /\left(\left(S_{i i}+S_{a a}+2 S_{i a}\right)-\frac{2 \chi_{i a}}{v_{0}}\left(S_{i i} \cdot S_{a a}-\left(S_{i a}^{2}\right)\right)\right)$

$i$ and $a$ stand respectively for the and deuterated macromonomer blocks of the backbone ( $i$ ) and for the protonated arms $(a)$. The $\chi_{i a}$ parameter, further abbreviated as $\chi$, now is an 
effective Flory-Huggins parameter and takes into account the interaction between the two components including the functional groups. As will be seen below also architectural and microstructural effect will contribute to the $\chi$ parameter. $v_{0}$ is the monomeric volume of the PBO unit. $\Delta \rho^{2}$ is the contrast factor and $N_{A}$ is Avogadro's number. The method used to calculate the contributions to the structure factor in the multiblock case as depicted in Fig.1 considers the system as composed of blocks each containing only monomers of one type. Considering a supramolecular comb-like block copolymer and assuming Gaussian statistics in the melt the unperturbed form factors in Eq. [2], which enter the partial structure factor in the RPA expression are written as: ${ }^{14}$

$$
\begin{aligned}
& \mathrm{P}_{i i}=J\left(n_{i}\right) \cdot N_{i}+H\left(n_{i}\right)^{2} \cdot \sum_{k=1}^{N_{i}-1}\left(N_{i}-(k+1)\right) \cdot G\left(n_{i}\right) \\
& \mathrm{P}_{a a}=J\left(n_{a}\right) \cdot N_{a}+H\left(n_{a}\right)^{2} \cdot \sum_{k=1}^{N_{a}-1}\left(N_{a}-k\right) \cdot G\left(n_{i}\right) \\
& \mathrm{P}_{i a}=2 \cdot H\left(n_{i}\right) \cdot H\left(n_{a}\right) \cdot \sum_{k=1}^{N_{a}}\left(N_{a}-k\right) \cdot G\left(n_{i}\right)
\end{aligned}
$$

where

$$
\begin{aligned}
& J(n)=\frac{2}{(X \cdot n)^{4}}\left(e^{-(X \cdot n)^{2}}-1+(X \cdot n)^{2}\right) \\
& H(n)=\frac{1}{(X \cdot n)^{2}}\left(1-e^{-(X \cdot n)^{2}}\right) \\
& G(n)=e^{-(X \cdot n)^{2}} \\
& X=\left(q \cdot l_{s t}\right)^{2} / 6
\end{aligned}
$$

$N_{i}$ is the total number of blocks of length $n_{i}$ in the backbone and $N_{a}$ corresponds to the number of arms of length $n_{a}$ attached and $l_{s t}$ is the statistical segment length per monomer. $J(n)$ corresponds to the self-term for each block type and is represented by the Debye function. With $H(n)$ the correlation between monomers belonging to different blocks is handled, while the correlation between non-adjacent blocks is accounted for the spacer term $G(n)$. Similar as in the linear case the sensitivity of the approach is high at low-to-intermediate arm association degrees.

The definition of the partial structure factors is:

$$
\begin{aligned}
& S_{a a}=\phi_{a b} \cdot v_{0} \cdot\left(N_{a} \cdot n_{a}\right) \cdot P_{a a} \\
& S_{i i}=\phi_{i} \cdot v_{0} \cdot\left(N_{i} \cdot n_{i}\right) \cdot P_{i i} \\
& S_{i a}=\sqrt{\phi_{a b} \cdot \phi_{i} \cdot\left(N_{i} \cdot n_{i}\right) \cdot\left(N_{a} \cdot n_{a}\right) \cdot v_{0}^{2} P_{a a} \cdot P_{i i}}
\end{aligned}
$$


A peak results from the combination of the partial structure factors in Eq. [1]. Its position and the intensity depend on the correlation between blocks with different scattering length density and are therefore correlated to the average aggregation number and the size of the correlation block $a$...i...a (Fig.1). The case of a random blend of linear homopolymers, which arises from the disconnection of the arms from the backbone, is recovered when the cross-term $S_{i a}$ is set to zero. A non-zero interaction term in a non-ideal mixture yields an additional scattering contribution as defined by the RPA expression and has to be considered as well. Fig.3 shows the effect of the length of arms (a) and of their number density (b) on the scattering profile of the block copolymer. The maximum aggregation number in this system depends on the density of functional groups attached to the backbone and in our case corresponds to 15 thymine groups per chain. Thus, the full association can lead ideally to the formation of a 15-armed block copolymer. The spatial extension of the inner deuterated block between adjacent thymine anchors - taken equidistant - in the supramolecular comb is a function of the aggregation number and limited by the backbone functionality. 

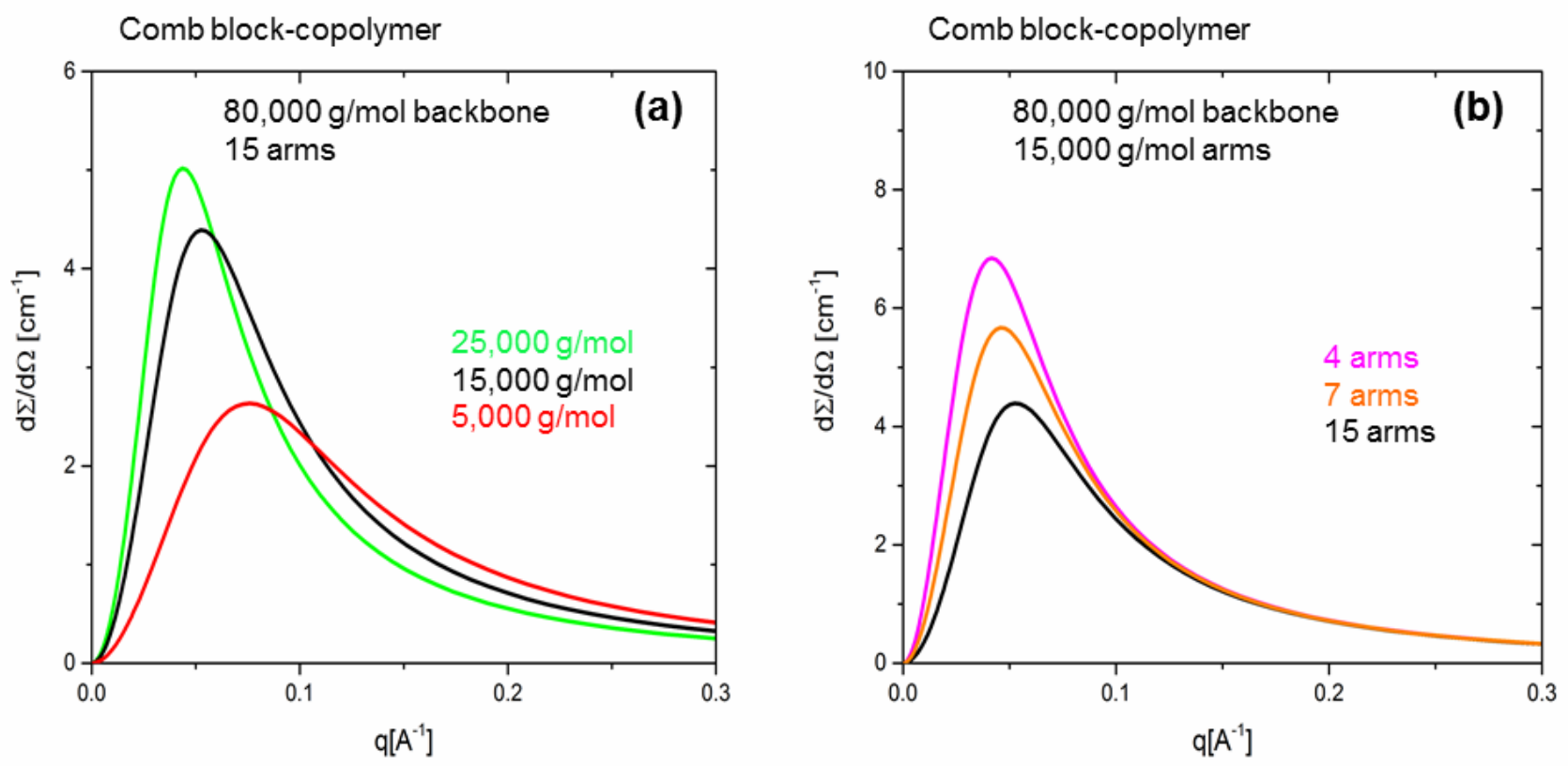

Figure 3: Effect of the arm size on the RPA function (a). Effect of the number of arms on the RPA peak intensity and position (b).

In the particular case of a supramolecular system the block copolymer, however, is always in equilibrium with free short chains. The RPA expression was therefore adapted to the case of a multiblock copolymer/linear homopolymer mixture. The additional free components, contributing to $S_{a a}$, leads to the finite scattering at low q due to the mixing effect and we assume that the contribution to the forward scattering derives only from the short hydrogenous chains. Free or non-functionalized backbones are neglected since it is highly unlikely that the long backbone does not carry any functional group at all.

Eq. [3] is therefore modified as:

$$
\begin{aligned}
& S_{a a}=\phi_{a b} \cdot v_{0} \cdot\left(N_{a} \cdot n_{a}\right) \cdot P_{a a}+S_{a} \\
& S_{i a}=\sqrt{\phi_{a b} \cdot \phi_{i} \cdot\left(N_{i} \cdot n_{i}\right) \cdot\left(N_{a} \cdot n_{a}\right) \cdot v_{0}^{2} P_{a a} \cdot P_{i i}} \\
& \text { where } \quad S_{a}=\phi_{a f} \cdot v_{0} \cdot n_{a} \cdot J\left(n_{a}\right)
\end{aligned}
$$


Therefore, the total volume fraction of arms in Eq. [4] is split in two components $\phi_{a b}, \phi_{a f}$, related to the volume fraction of arms correlated i.e. bonded (b) and uncorrelated i.e. free (f) with respect to the deuterated backbone such that the mass balance is respected. In the RPA model used to describe the data, $\phi_{a b}, \phi_{a f}, \chi$ and the effective statistical segment length $l_{s t}$ are the refining parameters while the number of monomers and the contrast according to the chemical characterization are kept constant at each temperature. The average aggregation number $\left(N_{a}\right)$ is directly related to the volume fraction of bonded arms. The maximum aggregation number $\left(N_{\max }\right)$ is related to the backbone functionalization as well as the stoichiometric ratio between arms and backbone. The mass balance we use was:

$\phi_{a b}=\phi_{a} \cdot(1-x)$

$\phi_{a f}=\phi_{a} \cdot x \quad N_{a}=\left(\phi_{a b} \cdot N_{\max }\right) / \phi_{a}$

The factor $x$ relates the fraction of free arms to the total volume fraction $\phi_{a}$

The contribution of the non-associating short chains weighted with the respective volume fraction $\phi_{a f}$ depends on the temperature: at higher temperature the fraction of uncorrelated $a$ $(H)$ and $i(D)$-blocks increases at the expense of the block copolymer. Fig.4 represents the RPA prediction for a blend homopolymer/copolymer, showing the contribution of the nonassociating chain on the scattering function between the two limits of the fully associating system, i.e. taking into account the functionality of the backbone d-PBO thy-80k and the inactive blend. The effect of the arm molecular weight was also examined. Furthermore, the effect of a $\chi$ parameter on the scattering intensity is included in Fig.4 (dashed lines) where a typical value of the interaction parameter for miscible blends, comparable with the $\chi$ parameter found for blends of functionalized components, (vide infra) was assumed. 

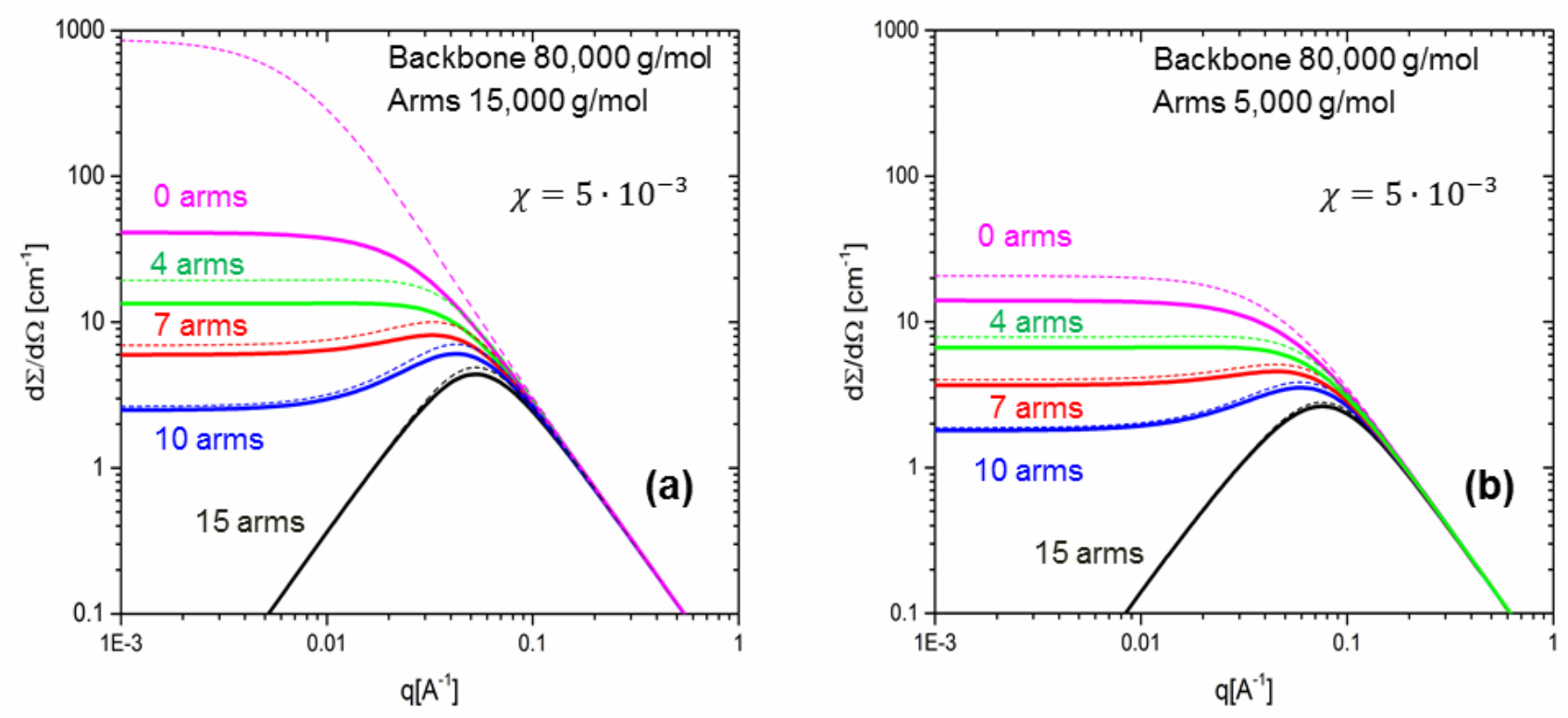

Figure 4: Theoretical scattering function for a homopolymer/copolymer blend from the fully associating (15 arms) to non-associating limit (0 arms) for different arm length and effect of $\chi$ parameter (dashed lines) on the scattering intensity. $15000 \mathrm{~g} / \mathrm{mol}$ arms (a) $5000 \mathrm{~g} / \mathrm{mol}$ arms (b) 


\section{Results}

Table 1. Composition of the samples

$\begin{array}{lll} & \text { Components } & \begin{array}{c}\text { Molar ratio } \\ \text { (Functional groups) }\end{array} \\ \text { S1 } & \text { d-PBO thy-80k/PBO 15k-DAT } & 1: 0.5 \\ \text { S2 } & \text { d-PBO thy-80k/PBO 15k-DAT } & 1: 1 \\ \text { S3 } & \text { d-PBO thy-80k/PBO 15k-DAT } & 1: 1.5 \\ \text { S4 } & \text { d-PBO 80k/PBO 15k } & 1: 0.5 \\ \text { S5 } & \text { d-PBO 80k/PBO 15k } & 1: 1 \\ \text { S6 } & \text { d-PBO 80k/PBO 15k } & 1: 1.5 \\ \text { S7 } & \text { d-PBO thy-80k/PBO 5k-DAT } & 1: 0.5 \\ \text { S8 } & \text { d-PBO thy-80k/PBO 5k-DAT } & 1: 1 \\ \text { S9 } & \text { d-PBO thy-80k/PBO 5k-DAT } & 1: 1.5\end{array}$

4.1. Small Angle Neutron Scattering: SANS results for functionalized mixtures of $H(a)$ and D (i)components are presented and compared with the SANS data of a random blend of inactive D (i) and $\mathrm{H}(a)$-blocks at different stoichiometric ratios and different temperatures. The molar ratio for each blend is referred to the functional groups. A power law $\mathrm{I}(q) \sim q^{-4}$ observed at low $q$ due to small voids present in the samples and the high contrast between these and the deuterated components, was fitted along with RPA model and then subtracted from the experimental data. Short chains with different molecular weight were used in order to study the effect of the arm length and their concentration on the scattering function of the supramolecular system (Table 1 ). The data are reported in absolute units $\left[\mathrm{cm}^{-1}\right]$ and the qdependence at high scattering vector displays a power law of -2 characteristic for Gaussian chains in both cases. The blend of linear chains without functional groups (S5) shows a Debyelike behavior, while in the presence of the hydrogen bonding groups (S2) a correlation hole is visible in the scattering function (Fig.5(a)). In the scattering profile of the functionalized mixture the correlation peak is observed at intermediate $q$, even though the peak does not follow the pronounced correlation hole as expected for a pure diblock comb-copolymer. The non-zero intensity at $q=0$ is related to the presence of non-associating chains. NMR investigations show ${ }^{9}$ that a small fraction of the hydrogenous arms is not functionalized and, depending on the stoichiometric ratio, not the whole fraction of short chains can thus be correlated to the 
backbone. The aggregation number as well as the position of the correlation peak depend on the volume fraction of bonded arms and backbone in the mixtures as shown in Fig.5(b).
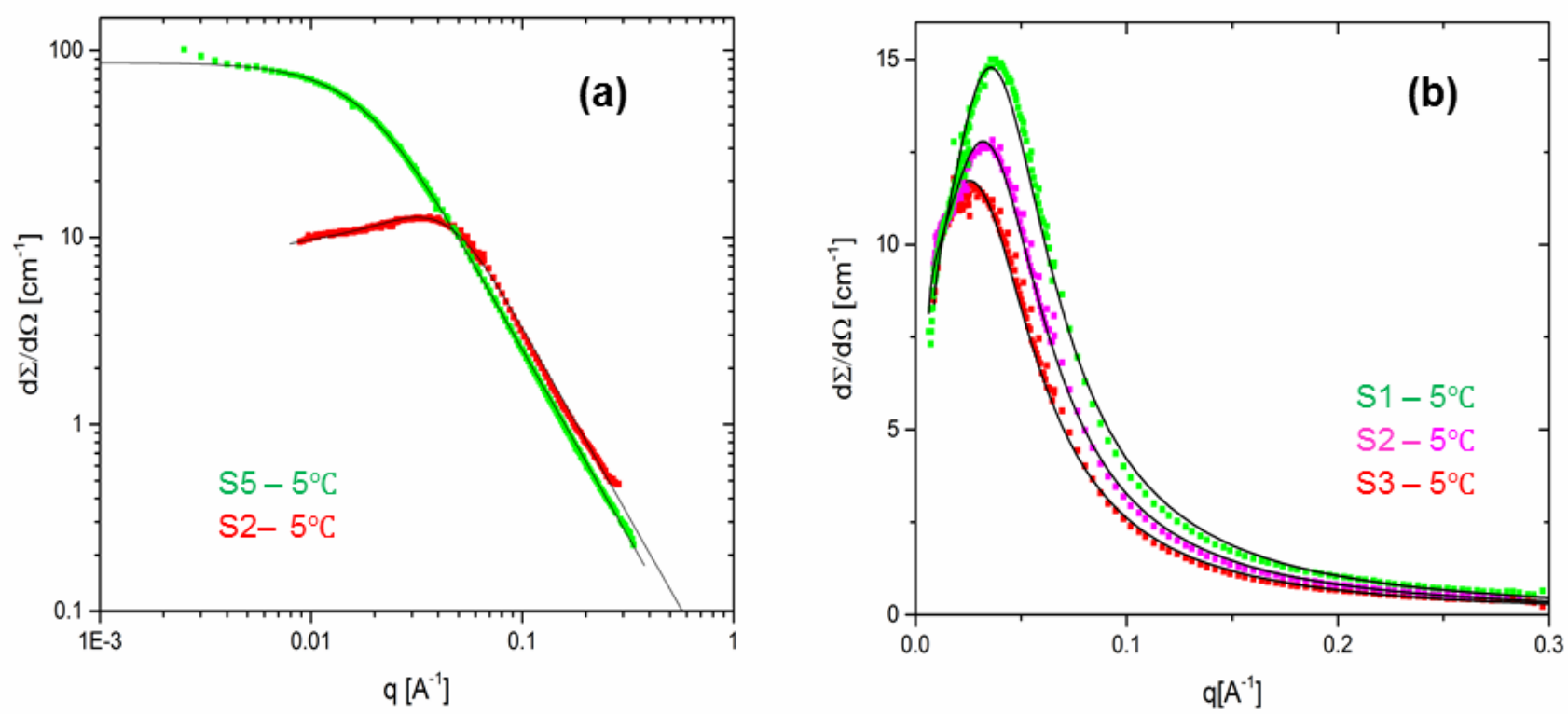

Figure 5: Equimolar non-functionalized and functionalized blends of $\mathrm{H}$ - and $\mathrm{D}$-components (KWS2 data). The stoichiometry of the samples is in the best-possible agreement (a).Blends of functionalized components at different stoichiometric ratios of functional groups (KWS-2 data) (b).

The effect of the temperature on the association degree was also studied in this experiment. Fig. 6 clearly shows that an increase of the forward scattering at low $q$ is observed by increasing the temperature from $5{ }^{\circ} \mathrm{C}$ to $100^{\circ} \mathrm{C}$. However, the correlation peak is still visible in the scattering function at $100{ }^{\circ} \mathrm{C}$, showing that the equilibrium between the associating system and the free short chains did not vanish fully at this elevated temperature and still associated species is present. 


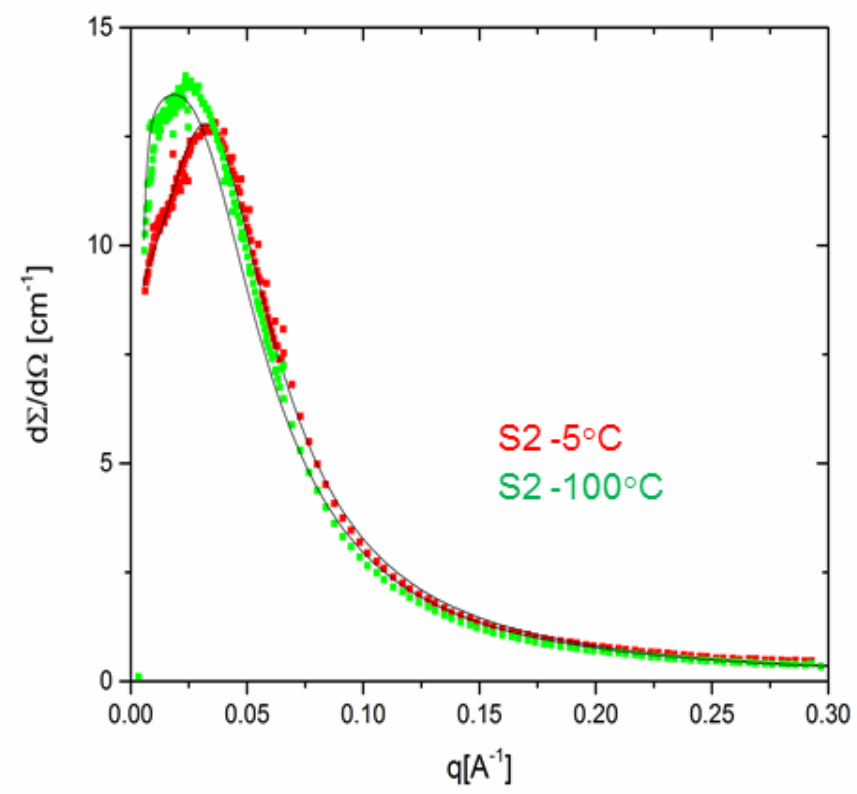

Figure 6: Effect of the temperature on the scattering intensity and RPA fit.

The experimental $\chi$ parameter fitted by means of the RPA model, restricted only to two components, takes into account the effective interaction between the deuterated and hydrogenous components. The effect of the functional groups on the interaction parameters is shown in Fig. 7 where the average $\chi$ is reported as function of temperature. Typical values of isotopic $\chi$ parameter for blends of deuterated and hydrogenous linear polymer are reported in literature,$^{15}$ as well as the effect of the architecture on the interaction parameter in blends of linear and branched polymers. ${ }^{8,16}$ In the absence of supramolecular bonding groups $\chi$ is $\sim 10^{-3}$ for $\mathbf{5 5}$, which is slightly higher than for other typical isotopic mixtures. In the case of the functionalized blends (S2) the increase of the average interaction parameter by a factor of three is therefore related to the change of architecture induced by the association of the components into a comb-like structure. This contribution leads to a considerable enhancement of the interaction parameter depending on the functionality and on the volume fraction of the branched component, as already reported in literature. ${ }^{15,16}$ In the present case we point out that already the non-functionalized mixture is affected by the $C_{6}$ alkyl side chains of the 1,2epoxy-7-octene comonomer (Fig.2) and therefore a higher $\chi$ may result. Fig.7 shows the averaged $\chi$ values for the respective blends at different stoichiometric ratios, since only negligible variations of the $\chi$ parameter with composition within statistical uncertainties were 
observed. The actual values of the $\chi$ parameters obtained for the functionalized and inactive blends are reported in Supporting Information. The temperature dependence is not significantly different in the two samples examined and suggests that the enthalpic contribution is probably entirely related to the miscible blend character rather than to the architecture effect. $^{15 b}$

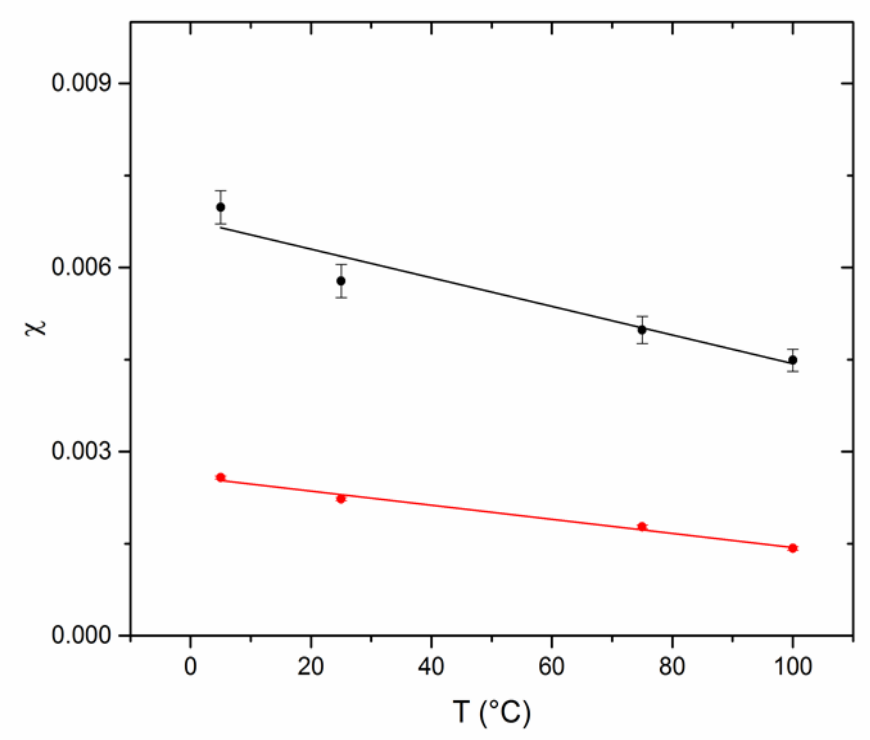

Figure 7: Average Interaction parameter for the functionalized blends (S1, S2, S3) (in red) and for the inactive blends $(\mathbf{S 4}, \mathbf{S 5}, \mathbf{S 6})$ (in black) as function of the temperature 
Table 2. Fit Parameters using the RPA approximation at different T

\begin{tabular}{lcl} 
& \multicolumn{1}{c}{$N_{a}$} & $l_{s t}(\AA)$ \\
S1 & & \\
$5{ }^{\circ} \mathrm{C}$ & $4.40 \pm 0.04$ & $5.41 \pm 0.03$ \\
$25{ }^{\circ} \mathrm{C}$ & $4.25 \pm 0.05$ & $5.74 \pm 0.03$ \\
$75{ }^{\circ} \mathrm{C}$ & $3.68 \pm 0.05$ & $5.64 \pm 0.02$ \\
$100{ }^{\circ} \mathrm{C}$ & $3.22 \pm 0.07$ & $5.75 \pm 0.03$ \\
$\mathbf{S 2}$ & & \\
$5{ }^{\circ} \mathrm{C}$ & $7.07 \pm 0.07$ & $5.61 \pm 0.04$ \\
$25{ }^{\circ} \mathrm{C}$ & $7.03 \pm 0.1$ & $5.65 \pm 0.05$ \\
$75{ }^{\circ} \mathrm{C}$ & $6.28 \pm 0.08$ & $5.83 \pm 0.05$ \\
$100{ }^{\circ} \mathrm{C}$ & $4.84 \pm 0.04$ & $5.35 \pm 0.03$ \\
$\mathbf{S 3}$ & & \\
$5{ }^{\circ} \mathrm{C}$ & $7.5 \pm 0.1$ & $5.83 \pm 0.06$ \\
$25{ }^{\circ} \mathrm{C}$ & $7.7 \pm 0.1$ & $5.92 \pm 0.06$ \\
$75{ }^{\circ} \mathrm{C}$ & $7.36 \pm 0.09$ & $5.87 \pm 0.04$ \\
$100{ }^{\circ} \mathrm{C}$ & $6.95 \pm 0.08$ & $5.99 \pm 0.04$
\end{tabular}

Table 2 summarized the results of the RPA description for the functionalized blends d-PBO thy80k/PBO 15k-DAT at the different compositions. The statistical segment length $l_{s t}$ found is in good agreement with a reported value for PBO from literature. ${ }^{10}$ The average aggregation number $N_{a}$ found through the RPA model varies as expected as a function of the stoichiometric ratio between the functional groups and temperature. It was found that in the equimolar mixture and even with a stoichiometric excess of arms the average aggregation number did not exceed 7-8. The increase of the forward scattering by increasing the temperature in Fig. 6 is related to the increased fraction of non-correlated blocks but the average $N_{a g g}$ is just slightly affected by the temperature. A decrease of the $\chi$ parameter, observed with the increase of the temperature, affects the scattering intensity as well. We have shown before that the interaction parameter and the aggregation number influence the peak amplitude in opposite sense. Nevertheless, the effect of $\chi$ is limited to the intermediate q scale while the latter is additionally related to the free arms volume fraction and therefore also the forward scattering is a sensitive descriptor. The determination of the average aggregation number by means of the RPA model is therefore only marginally affected by the influence of the interaction parameter. 
The effect of the size of the arms is evident in the experimental scattering curves (Fig.8). Here, experimental results obtained for representative $\mathbf{S 8 ,}$ which has $5 \mathrm{k}$ graft arms are shown. The $\chi$ parameter in this set of blends was found to be smaller. Again, the experimental scattering function does not show a clear correlation peak anymore as would be expected. Fig.8 shows the prediction of the scattering function for the two limits i.e. inactive blend and pure block copolymer as well as the experimental data and the fit function with the RPA model. The absence of a clear peak can be ascribed again to the contribution of free arms which strongly affects the scattering function in the $q$-range where the correlation peak occurs. The best fit with the RPA model yields a number of arms close to 7 at $5{ }^{\circ} \mathrm{C}$ for the equimolar blend, as found for the sample $\mathbf{S 2}$ with the same stoichiometric ratio but of triple arm length. Also in this case blends at different stoichiometric ratio backbone/arms were measured at different temperatures. Due to the interference of the free short arms with the correlation peak and to the small effect of the $\chi$ parameter, the temperature increase did not have a significant effect on the absolute intensity.

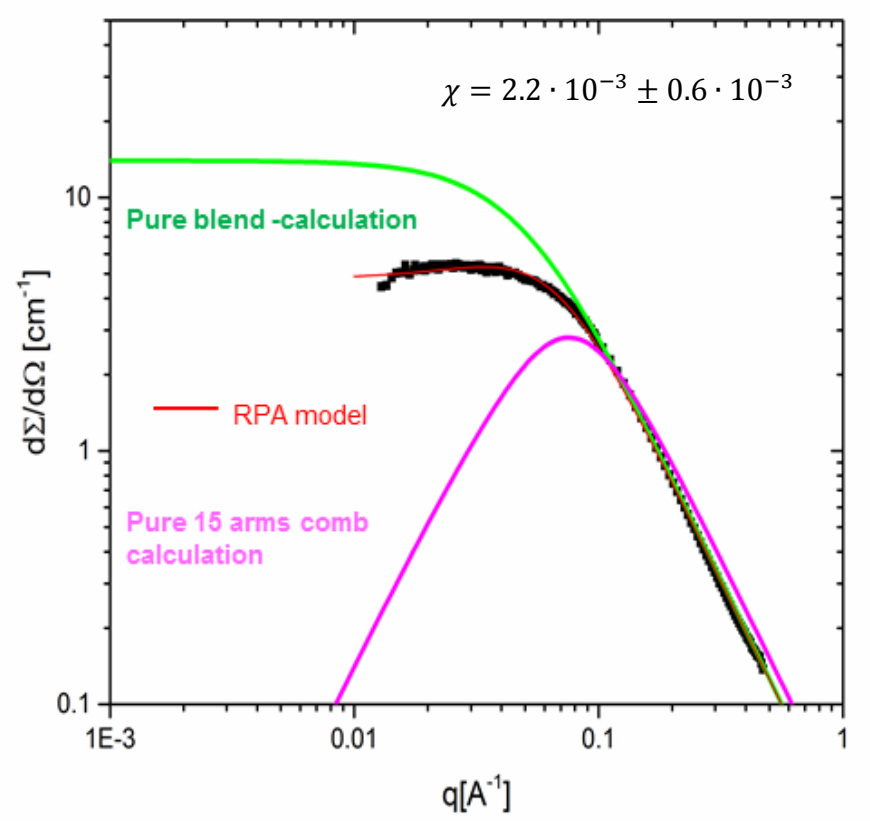

Figure 8: RPA prediction for the limit of an inactive blend and a block-copolymer with $5 k$ arms at $5^{\circ} \mathrm{C}$ (respectively green and magenta). Experimental results for $\mathbf{8 8}$ (black) and fit with RPA model (red) (V4 data). 
Association constant: The possibility to estimate the average aggregation number in the melt state as a function of temperature renders SANS as a unique probe of the equilibrium constant. The association constant $K_{a}$ was determined by the aggregation number found by using the RPA results. The equilibrium between open and closed state is well established:

thy + DAT $\leftrightarrows$ thy DAT

$K_{a}=\frac{[T H Y D A T]}{[T H Y] e q \cdot[D A T] e q}$

Thus, the average aggregation number allows the determination of the association constant directly in the melt. The value of $K_{a}$ at $25{ }^{\circ} \mathrm{C}$ for the equimolar blend corresponds to $\sim 30$ $\mathrm{L} \mathrm{mol}^{-1}$. This is much lower than in a similar approach by SANS on PEG ${ }^{7}$ using the same thy DAT complex, where the association constant for the hetero-complementary groups was estimated in the melt at $25^{\circ} \mathrm{C}$ as $\sim 300 \mathrm{~L} \mathrm{~mol}^{-1}$. The experimental limit of aggregation number $(\sim 7)$, independent of the size of the arms, in our present investigation may be related to the availability of the groups grafted on the backbone. The thymine groups are indeed randomly distributed along the backbone and probably not all are fully accessible for e.g. sterical reasons. The $K_{a}$ calculated for $\mathbf{S 2}$ is reported in Fig. 9 as function of temperature. These values were estimated, in the present work, assuming that all the 15 groups per backbone chain are equally accessible. In order to estimate the actual maximum functionality of the backbone, we have used $K_{a}$ of $\sim 300 \mathrm{~L} \mathrm{~mol}^{-1}$ determined for the very related PEG system in reference [7]. This assumption leads to a maximum of accessible bonding sites of $\sim 9$ instead of 15 , which agrees better with our average aggregation number of $\sim 7$. 


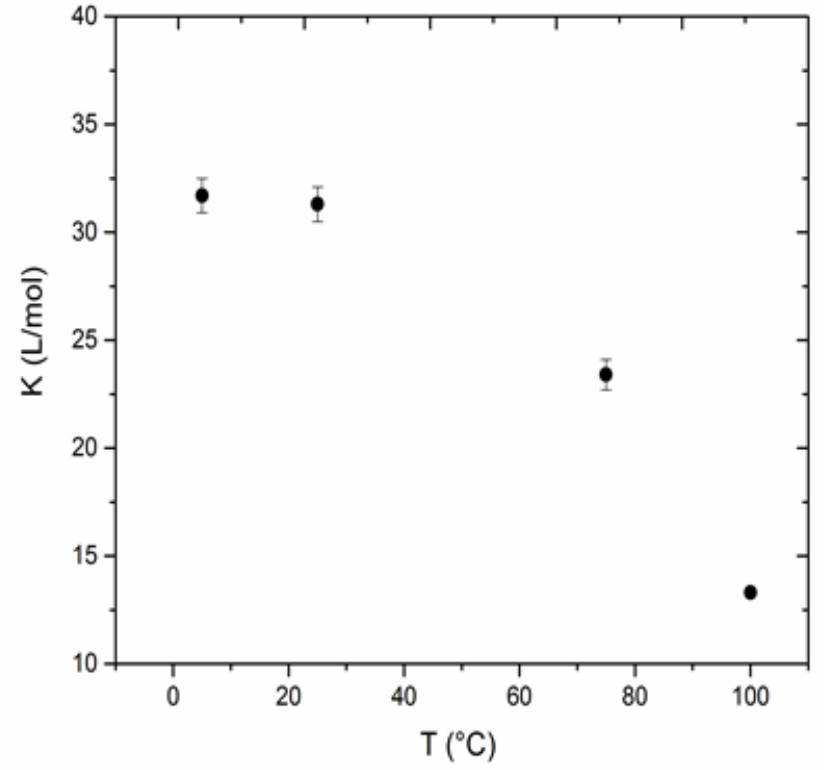

Figure 9: Experimental association constant for equimolar blend as a function of temperature. 
Table 3. Sample classification

\begin{tabular}{|l|l|l|}
\hline Pure components & & \\
\hline d-PBO thy-80k & & \\
\hline PBO 15k-DAT & & \\
\hline PBO 5k-DAT & & \\
\hline Blends & Molar ratio & $\phi$ (d-PBO thy-80k) \\
\hline d-PBO thy-80k/PBO 15k-DAT & $1: 0.5$ & 0.26 \\
\hline d-PBO thy-80k/PBO 5k-DAT & $1: 0.5$ & 0.68 \\
\hline d-PBO thy-80k/PBO 15k & $1: 0.5$ & 0.26 \\
\hline d-PBO thy-80k/PBO 5k & $1: 0.5$ & 0.68 \\
\hline
\end{tabular}

4.2. Linear Rheology: Linear Rheology experiments were carried out with both the functionalized precursors and the inactive blends in order to investigate the effect of the supramolecular bonds on the macroscopic dynamics of the polymer systems. Fig.10 shows the storage $G^{\prime}(\omega)$ and loss $G^{\prime \prime}(\omega)$ moduli for functionalized components vs $\omega$ ), reduced to $T_{0}=$ $25^{\circ} \mathrm{C}$. For all pure functionalized components (d-PBO thy-80k, PBO 15k-DAT, PBO 5k-DAT) the data exhibit clear low frequency $\omega^{1}$ and $\omega^{2}$ dependences for $G^{\prime \prime}$ and $G^{\prime}$ respectively, characteristic of viscous flow. Furthermore, a well-pronounced plateau region in the intermediate frequency range was observed for the entangled thy-modified backbone. The entanglement plateau modulus of $\sim 0.30 \mathrm{MPa}$ estimated for PBO corresponds to an average mass $M_{e}$ between entanglements of about $8,000 \mathrm{~g} / \mathrm{mol} .{ }^{9,17}$ For PBO 15k-DAT endfunctionalized arms, also a small plateau can be detected, indicating a slightly entangled behavior which could appear if two chains each $2 M_{e}$ long entangle (Fig.10(a)). The 'plateau modulus' observed in these DAT -functionalized arms is therefore ascribed to the self association of the functional groups which leads to the formation of dimers. For PBO 5k-DAT (Fig.10 (b)) the molecular weight is lower than the estimated $M_{e}$ and therefore also any resulting dimers still show a Rouse-like behavior. As reported in a previous study no sign of self - association was revealed for the thymine functionalized backbone. Instead, a more pronounced effect of the functionalization with DAT groups was observed for the short chains ${ }^{9}$. In the present study, while the non - functionalized precursor PBO 15k exhibit a Rouse - like behavior, the data obtained for PBO 15k-DAT can be described by a combination of a Rouse and BSW model (vide infra) in order to take into account the equilibrium between the non associated short chains and the dimers (see Supporting Information). The system behaves as a blend of $\sim 40 \%$ entangled dimers $\left(M>M_{C} \sim 2 M_{e}\right)$ and $\sim 60 \%$ non - entangled unimers $\left(M<M_{C}\right)$. This approach allows an estimation of the association constant for the DAT - DAT 
association of the order of $5 \mathrm{~L} \mathrm{~mol}^{-1}$ at $-25^{\circ} \mathrm{C}$. The already small value of the constant at low temperature, allows us to neglect the self - association mechanism for the DAT - functionalized component especially in the temperature range of the SANS experiment.
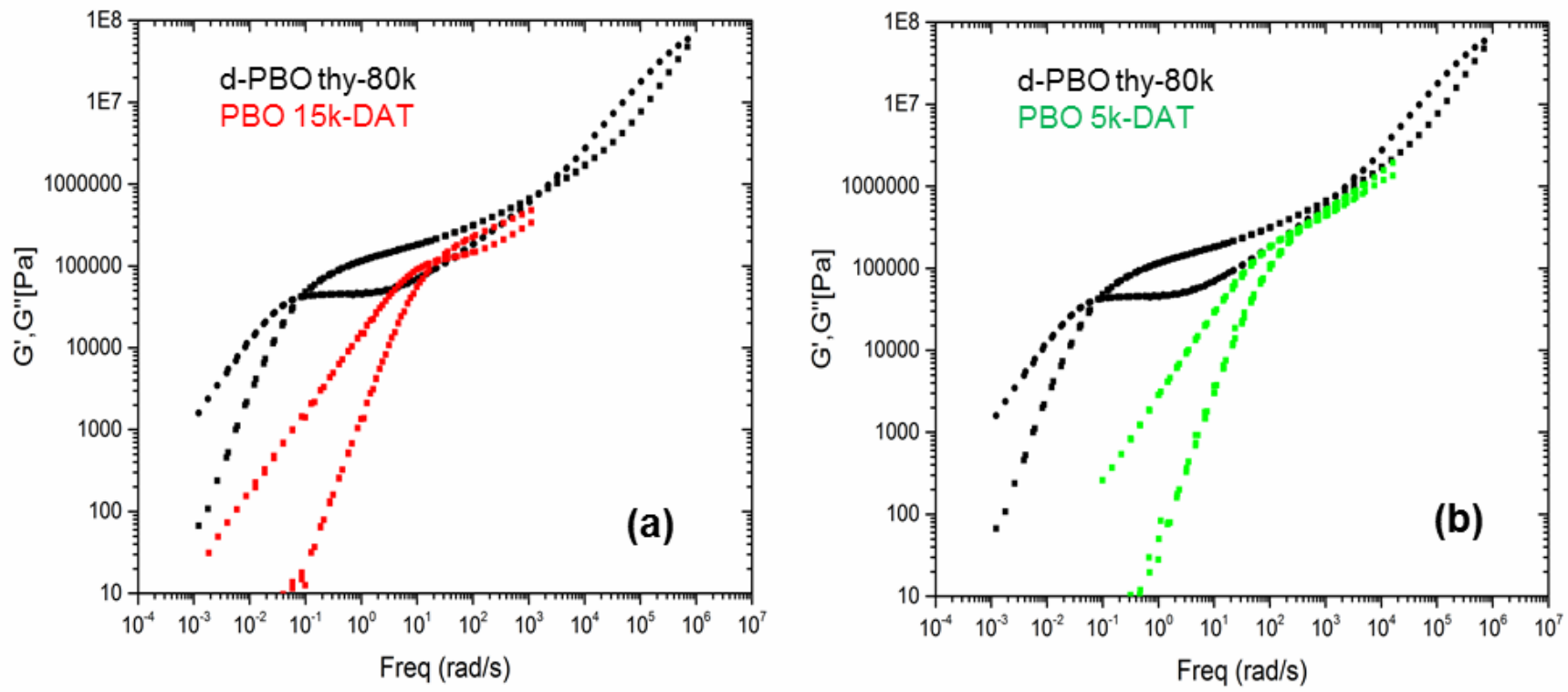

Figure 10: Storage and loss moduli for $\boldsymbol{d}-\mathrm{PBO}$ thy-80k and PBO15k-DAT (a) and PBO 5k-DAT (b).

For the blends the situation is more complex. Fig.11 shows data obtained for the associating and semi-functionalized blends of the long backbone in combination with PBO 15k-DAT and PBO 5k-DAT. In semi-functionalized blends, d-PBO thy-80k was used instead of pure d-PBO $\mathbf{8 0 k}$ as it does not show any signs of homo-association. ${ }^{9}$ In the first case the blend of semifunctionalized components (red below) shows a close to Rouse-like behavior at long times typical of a non-entangled polymer system. The Rouse-like behavior at intermediate frequency $\left(\sim \omega^{1 / 2}\right)$ is due to the dilution of the entangled chain tube or entanglement network with the shorter unentangled chains. This strong dilution of the backbone was observed only in combination with the higher molecular weight arms. We note that such a Rouse behavior of an entangled chain was also observed in studies of the single chain dynamic structure factor from NSE on a long polyethylene chain diluted with short oligomers, from which the Rouse monomeric friction coefficient of the short matrix could be determined. ${ }^{18}$ In d-PBO thy$\mathbf{8 0 k / P B O ~ 1 5 k}$ the diluted tube diameter becomes comparable with the end-to-end distance of the backbone and this explains the Rouse-like behavior observed for the inactive blend. In the 
second case $\mathbf{d - P B O}$ thy-80k/PBO 5k, due to the low short chains volume fraction, the tube dilution of the entangled backbone is only partial. The system then retains the typical dynamics of an entangled polymer chain but is accelerated compared to the pure backbone. In both cases the storage modulus of the backbone in the in-active blend was observed to be related to the backbone volume fraction by the equation $G_{N}(\phi)=G_{N} \phi^{\alpha+1}$ where the exponent $\alpha$ is assumed to be comprised between 1 and 4/3. ${ }^{19}$
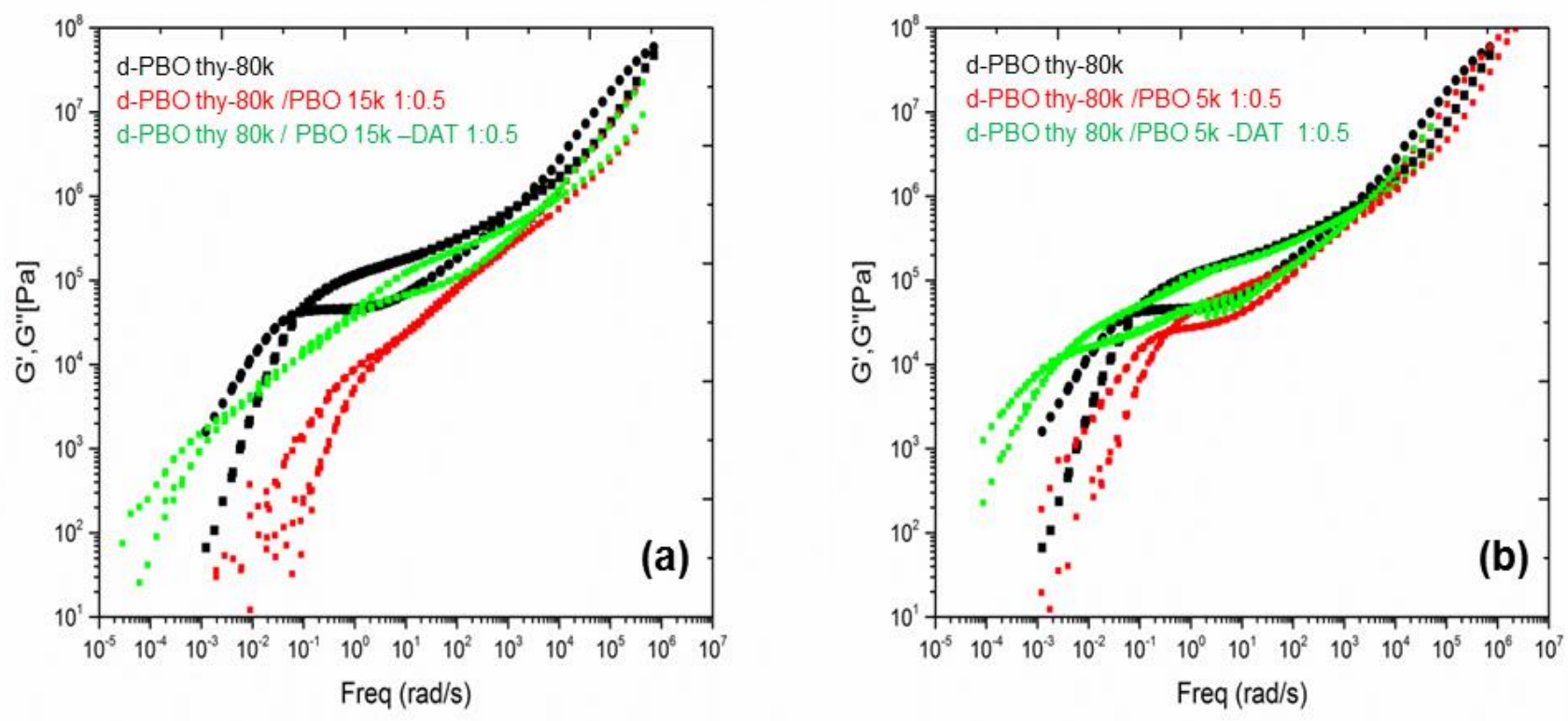

Figure 11: Storage- and loss moduli for functionalized backbone (black), functionalized blend (green), non-functionalized blend (red) for combinations d-PBO80k/PBO15k (a) and d-PBO80k/PBO 5k (b).

A totally different dynamics is observed in the blend of functionalized components with the same stoichiometric ratio (d-PBO thy-80k/PBO 15k-DAT and PBO thy 80k/PBO 5k-DAT). Since SANS on blends of functionalized components clearly proved the transient branched architecture, signatures of them are expected as well in the linear rheology. With respect to this a plateau region in $G^{\prime}(\omega)$ occurs at intermediate frequency which is associated with an additional relaxation mechanism in $G^{\prime \prime}(\omega)$ correlated with the association/dissociation of the components of the blend. This process leads to the formation of a comb-like structure in equilibrium with a fraction of non-associating free arms within the lifetime of the transient 
bonds. The plateau modulus for the functionalized blend is comparable with the modulus of the undiluted backbone and it points to a hetero-complementary association of the transient groups. At low frequencies the dynamics is again dominated by the diluted long chain as the transient bonds are not effective anymore. The storage modulus at low frequency is comparable with the one observed for the semi-functionalized blend proving that at times longer than the transient bond lifetime the system behaves like a simple blend (Fig.11, green in the middle). The viscoelastic behavior is therefore the result of two competing effects. First, the delay in the terminal relaxation of the long chain in the supramolecular comb is due to the additional process arising from the association/dissociation of the components of the blend and is the classical signature of covalent but branched polymers for which a hierarchical relaxation is expected. The association not only increases the effective friction but also slightly interferes with the backbone dynamics, admittedly less than in the covalent comb, even in the linear rheology case. On the other hand, unavoidable dilution effects contribute to the acceleration of the terminal relaxation of the backbone. Two relaxation processes are thus clearly distinguishable in the blends of thy and DAT-modified PBO chains. The data seems to obey to the time-temperature superposition principle over the whole frequency range. For the pure backbone and the inactive blends (respectively in black, orange and green) the temperature dependence of the shift factor is well described by the WLF equation and is comparable in the three cases (Supporting Information). Note that the shift factors are those of a blend. A difference in the temperature dependence was observed in the case of the functionalized blends due to the additional process related to the transient bonds. The effect of the transient bonds on the thermo-rheological behavior of telechelic polymer systems has been reported in literature. ${ }^{20}$ The different temperature dependence of the relaxation processes involved can originate from a thermo-rheological complexity in the frequency range where the reversible bonds are active. In our case it was observed that d-PBO thy-80k /PBO 15k DAT still conform to the WLF equation although the dynamics is dominated by two well-separated relaxation processes. This indicates a comparable temperature dependence of the two mechanisms involved or a vanishing effect. A deviation from the WLF behavior was observed for d-PBO thy80k /PBO 5k DAT which may show a small thermo-rheological complexity at the time scale of the transient bonds dissociation. Although the two associating blends examined involve the same relaxation mechanisms, the composition and the dilution effect are different in the two cases. The more pronounced effect of the transient bonds on the dynamics, in the case of $\mathbf{d}$ PBO thy-80k/PBO 5k-DAT, could be explained by considering the more effective separation of the bond lifetime and the backbone terminal relaxation due to a lower dilution factor. The small difference in temperature dependence of the two mechanisms involved is therefore more evident in the last case.

In the limiting case of a permanent comb polymer (or transient with long lifetime exceeding the terminal relaxation time), a hierarchical relaxation scheme would be expected due to the 
retraction of the arms and consequently to the delayed relaxation of the diluted backbone. ${ }^{21}$ The additional relaxation mechanism, resulting from the formation/breakage of the transient bonds, could be approximated by a Maxwell element with a characteristic time $\left(\tau_{b}\right)$ corresponding to the transient bond lifetime. Such a description of the data was attempted by using the phenomenological $\mathrm{BSW}^{22}$ model in combination with a Maxwell model taking into account the faster relaxation process in the relaxation spectrum.

The viscoelastic moduli $G^{\prime}$ and $G^{\prime \prime}$ can be obtained by the Fourier transform of a continuous relaxation spectrum:

$$
\begin{aligned}
& G^{\prime}(\omega)=G_{\infty}+\int_{0}^{+\infty} H(\tau) \omega^{2} \tau^{2} /\left(1+\omega^{2} \tau^{2}\right) d(\ln \tau) \\
& G^{\prime \prime}(\omega)=\int_{0}^{+\infty} H(\tau) \omega \tau /\left(1+\omega^{2} \tau^{2}\right) d(\ln \tau)
\end{aligned}
$$

where $G_{\infty}$ is the modulus at infinitely low frequencies and $H(\tau)$ is a continuous not priorly known relaxation spectrum.

In the BSW model the relaxation spectrum is expressed as:

$$
\begin{array}{ll}
H(\tau)=H_{e} \tau^{n e}+H_{g} \tau^{-n g} & \tau_{0}<\tau<\tau_{\max } \\
H(\tau)=0 & \tau>\tau_{\max }
\end{array}
$$

where $H_{e}$ and $H_{g}$ define the entanglement and the glassy transition zone respectively. $n_{e}$ and $n_{g}$ are identified as the slopes in the loss modulus.

A tentative fit of the storage modulus as well as the two components of the model is reported in the Supporting Information, but was found to capture only part of the spectrum. In the case of d-PBO thy-80k/PBO 5k-DAT, the short component of the blend is not entangled and, as observed by the SANS experiment, the aggregation of the short chains to the backbone only has a marginal effect on the microscopic structure. The effect of the association/breakage process 
is nevertheless evident in the macroscopic dynamics of the mixture. A small thermo-rheological complexity is observed in the frequency range of the supramolecular association. Its effect on the WLF master curve can be seen in the terminal frequency region where the two-power-law BSW model does not describe the data well. A molecular rheological description in terms of a time marching algorithm is, however, underway and no further interpretation of the deviation is pursued at the present stage.

The elastic contribution to the plateau modulus, corresponding to this Maxwell-like process within experimental uncertainties, is comparable in the two different blends with the same stoichiometric ratios and was estimated as $\sim 0.04 \mathrm{MPa}$. The characteristic time of this process is function of the component volume fraction. The lifetime, associated with the second relaxation peak in $G^{\prime \prime}(\omega)$, observed in the blends of the backbone with $15 \mathrm{k}$ and $5 \mathrm{k}$ arms is $\sim 0.5 s$ and $\sim 1 s$ respectively due to a slightly different composition and may depend on a different friction of the two blends. A comparison can be made to a recent NSE study on the microscopic dynamics of the thymine and diaminotriazine association in a PEG matrix. Here, a bond lifetime of the order of $\sim 800 n s$ at $80^{\circ} \mathrm{C}^{23}$ was derived. For our PBO system which is closely related to the PEG backbone, a lifetime of the bonds of $\sim 600 \mathrm{~ns}$ is estimated at the same temperature using the experimental shift factors $\mathrm{a}_{\mathrm{T}}$. Since this involves an extrapolation over 10 orders of magnitude, however, only a very rough estimation of the lifetime can be achieved. In the same way rheological data reported in literature for bi-functional PnBA bearing thymine and diaminotriazine groups ${ }^{5 b}$ and referring to TTS using $T_{g}$ as the reference temperature, yield a characteristic lifetime for that system to be $\sim 100 \mathrm{~ns}$ at $80^{\circ} \mathrm{C}$. However, the same uncertainties apply. The present experimental time scales of $\sim 1 \mathrm{~s}$ at $-25^{\circ} \mathrm{C}$ can be exploited in mechanical probing in the order of the Hertz scale which would allow autonomous self-healing to happen in combination with a permanently crosslinked network.

Although rheology is strong in identifying the molecular dynamics of polymers the modulus sometimes is not detailed enough. In addition the dynamical-mechanical data were used to determine tentative relaxation spectra according to the method of Stadler and Bailly, ${ }^{24}$ which was found to provide the most accurate spectra among several methods tested. ${ }^{25}$ This method does not calculate the spectrum itself by determining different modes individually but describes the spectrum with a spline whose knots (as spectrum descriptors) are adjusted in logarithmized relaxation strength $\log \mathrm{H}$-position and $\operatorname{logarithmized}$ relaxation time $\log \tau$ to set up a continuous spectrum, discretized at 10 modes per decade. This is sufficient to describe all except for the most sharp spectra for which a higher mode density is necessary to obtain an accurate spectrum. Such continuous spectra were obtained for our data and the signature of the mechanisms emphasized. For the functionalized blends these relaxation spectra $H(\omega=1 / \tau)$ are included in Fig.12. It is clear that the dissipative maximum from the association-dissociation mechanism is a Maxwell-like process. The maxima occurring at low frequency are associated 
respectively to the Rouse time (Fig.12(a)) and to the disentanglement time (Fig.12(b)) of the diluted backbone. In Fig.12 (a) three peaks are visible in the relaxation spectrum. The low frequency range is dominated by the dynamics of the diluted backbone. It is not clear if the intermediate peak corresponds to a real relaxation process or whether it must be ascribed to an artifact. Since the former estimation of the relaxation spectrum is not univocal and the average of different solutions delivers the best approximation of the real spectrum artifacts cannot be excluded entirely. Therefore, the presence of a third peak in the spectrum could be related as well to a waviness of the experimental points at low frequency rather than to a real relaxation process. Moreover, this intermediate peak was not observed in the case reported in Fig.12(b), where the dilution factor is lower and the modulus observed at low frequency is higher. In any case the most significant result is the distinction in the relaxation spectra of a clear Maxwell-like process indicating an additional mechanism attributed to the transient bonds. For the sake of the present investigation the processes occurring at times longer than the characteristic time of the transient bonds are not relevant for the purposes of the description of the dynamics of the transient comb-like system. A fair-to-good agreement was found between the faster relaxation mechanism determined from the continuous relaxation spectrum and the characteristic time of the Maxwell element obtained through the description by using the combination of the BSW and Maxwell model. 

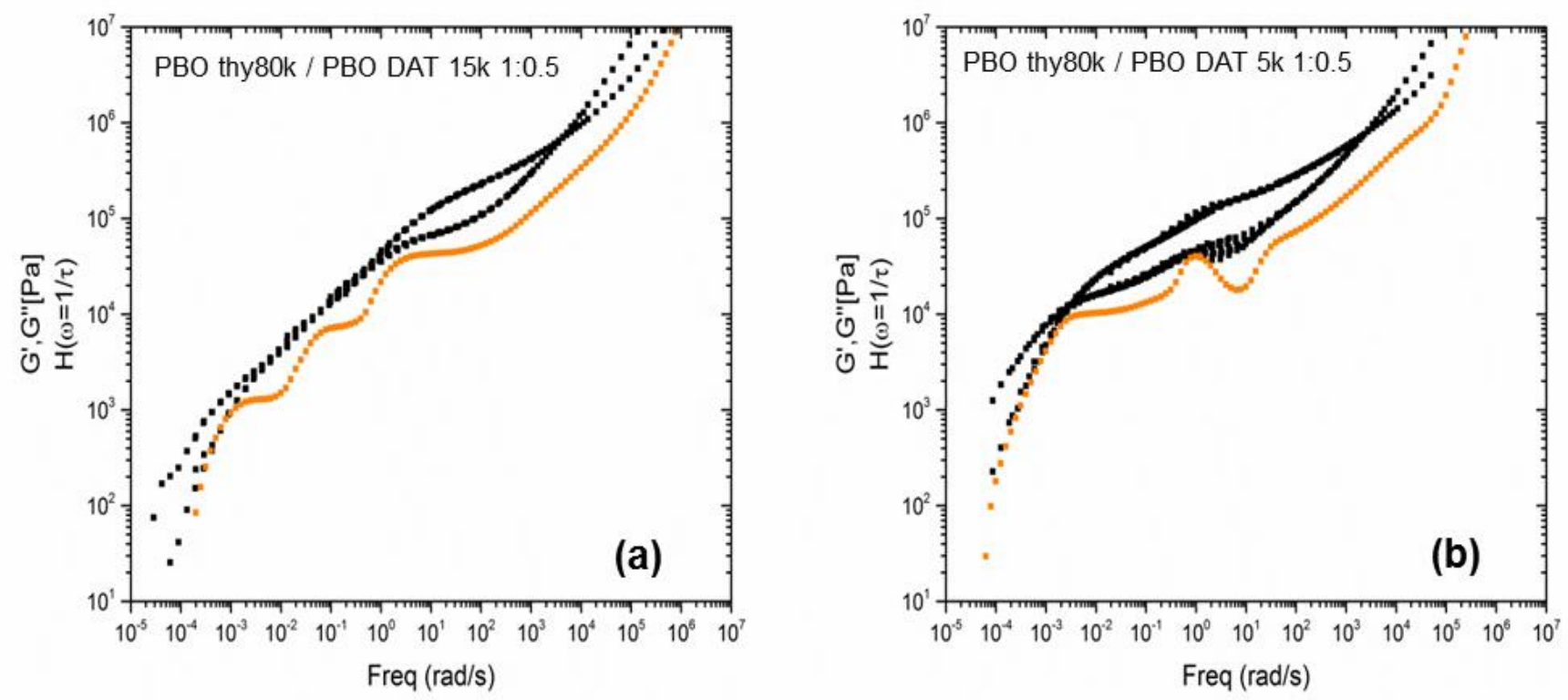

Figure 12: Storage and loss moduli (black) and relaxation time spectrum (orange) for d-PBO thy80k/PBO 15k-DAT (a) PBOthy 80k/PBO 5k-DAT (b). 


\section{Conclusions}

The microscopic and macroscopic investigation of the thymine- and diaminotriazine modified PBO chains in comparison with the simple blends of PBO based polymer chains reveal that the transient association of the hydrogen bonding groups has a strong effect on the microstructure and the dynamics of the polymer system. SANS and rheology experiments prove that the hetero-complementary association of the thymine- and diaminotriazine groups, leading to the formation of a transient comb-like structure, is dominant in the melt state. Self-complementary interactions do not yield contributions as experimentally shown. The results of the SANS measurements on functionalized blends show a clear correlation peak which is an indication of the hetero-complementary association and the formation of a transient diblock copolymer described by means of the RPA approximation. The hetero-complementary association was proven by the effect of the temperature on the scattering profile of the associating system. The average aggregation number and the association constant for the associating groups in the polymer matrix were determined as function of the temperature. The linear rheology experiments show a clear difference between the dynamics observed in the case of simple blends of linear chains and the same polymer blends functionalized with the associating groups. The evidence of two distinct relaxation processes attributed to the association/breakage of the transient bonds and to the terminal relaxation time of the long chain in the polymer blend, confirm the formation of a transient structure. The frequency dependence of the storage and loss moduli exclude the formation of unspecific clusters and confirm, as observed by SANS, the dominant hetero-complementary association. The analysis of the plateau moduli of the blends and pure components is not detailed, however, to allow a direct comparison and confirmation of the SANS results since the inclusion of the additional mechanism derived from the transient association is still required. The relaxation spectrum obtained from the viscoelastic moduli, although this is an ill-posed problem, allow the distinction of different relaxation process and clearly show a Maxwell-like process related to the transient bond association. Although a molecular description would be highly welcome to take into account the additional mechanism resulting from the association/breakage of the transient bonds, the rheology data corroborate the SANS evidence of a dominant hetero-complementary association, leading to the formation of a transient comb structure. 


\section{Acknowledgements}

The authors thank the financial support of the EU (ITN SASSYPOL 607602 Marie Curie Network). We acknowledge Dr. Nicole Lühmann for the help in the NMR analysis. 
${ }^{1}$ (a) Lewis, C. L; Stewart, K.; Anthamatten, M. The Influence of Hydrogen Bonding Side-Groups on Viscoelastic Behavior of Linear and Network Polymers. Macromolecules 2014, 47, 729-740.

(b) Hacklbusch, S.; Rossow, T.; van Assenbergh, P.; Seiffert, S. Chain Dynamics in Supramolecular Polymer Networks. Macromolecules 2013, 46, 6273-6286.

(c) Rossow, T.; Habicht, A.; Seiffert, S. Relaxation and Dynamics in Transient Polymer Model Networks. Macromolecules 2014, 47, 6473-6482.

(d) Hirschberg, J. H. K. K.; Beijer, F. H.; van Aert, H. A.; Magusin, P. C. M. M.; Sijbesma, R. P.; Meijer, E. W. Supramolecular Polymers from Linear Telechelic Siloxanes with Quadruple-Hydrogen-Bonded Units. Macromolecules 1999, 32, 2696-2705.

${ }^{2}$ (a) Cordier, P.; Tournilhac, F.; Soulié-Ziakovic, C.; Leibler, L. Self-healing and thermoreversible rubber from supramolecular assembly. Nature 2008, 451, 977-980.

(b) Herbst, F.; Seiffert, S.; Binder, W.H. Dynamic supramolecular poly(isobutylene)s for self-healing materials. Polym. Chem. 2012, 3, 3084-3092.

(c) Fox, J.; Wie, J. J.; Greenland, B. W.; Burattini, S.; Hayes, W.; Colquhoun, H. M.; Mackay, M. E.; Rowan, S. J. High-Strength, Healable, Supramolecular Polymer Nanocomposites. J. Am. Chem. Soc. 2012, 134, 5362-5368.

${ }^{3}$ (a) Binder, W.; Zirbs, R. Supramolecular Polymers and Networks with Hydrogen Bonds in the Main and Side-Chain. Adv. Polym. Sci. 2007, 207, 1-78.

(b) Pollino, J. M.; Weck, M. Non-covalent side-chain polymers: design principles, functionalization strategies, and perspectives. Chem. Soc. Rev. 2005, 34, 193-207.

(c) Wilson, A. J. Non-covalent polymer assembly using arrays of hydrogen-bonds. Soft Matter 2007, 3, 409-425.

Weck, M. Side-chain functionalized supramolecular polymers. Polym. Int. 2007, 56, 453-460.

(d) Ilhan, F.; Grey, M.; Rotello, V.M. Reversible Side Chain Modification through Noncovalent Interactions. "Plug and Play" Polymers. Macromolecules 2001, 34, 2597-2601.

${ }^{4}$ (a) Beijer, F.H.; Sijbesma, R. P.; Vekemans, J. A. J. M.; Meijer, E. W.; Kooijman, H.; Spek, A. L. HydrogenBonded Complexes of Diaminopyridines and Diaminotriazines: Opposite Effect of Acylation on Complex Stabilities. J. Org. Chem. 1996, 61, 6371-6380.

(b) Bellot, M.; Bouteiller, L. Thermodynamic Description of Bis-urea Self-Assembly: Competition between Two Supramolecular Polymers. Langmuir 2008, 24, 14176-14182.

(c) Pensec, S.; Nouvel, N.; Guilleman, A.; Creton, C.; Boué, F.; Bouteiller, L. Self-Assembly in Solution of a Reversible Comb-Shaped Supramolecular Polymer. Macromolecules 2010, 43, 2529-2534.

(d) Lou, N.; Wang, Y.; Li, X.; Li, H.; Wang, P.; Wesdemiotis, C.; Sokolov, A. P.; Xiong, H. Dielectric Relaxation and Rheological Behavior of Supramolecular Polymeric Liquid. Macromolecules 2013, 46, 3160-3166.

${ }^{5}$ (a) Brás, A. R.; Hövelmann, C. H.; Antonius, W.; Teixeira, J.; Raduescu, A.; Allgaier, J.; Pyckhout-Hintzen, W.; Wischnewski, A.; Richter, D. Molecular Approach to Supramolecular Polymer Assembly by Small Angle Neutron Scattering. Macromolecules 2013, 46, 9446-9454. 
(b) Herbst, F.; Binder, W. H. Comparing solution and melt-state association of hydrogen bonds in supramolecular polymers. Polym. Chem. 2013, 4, 3602-3609.

(c) Cortese, J.; Soulié-Ziakovic, C.; Leibler, L. Binding and supramolecular organization of homo- and heterotelechelic oligomers in solutions. Polym. Chem. 2014, 5, 116-125.

${ }^{6}$ (a) Yan, T.; Schroeter, K.; Herbst, F.; Binder, W. H.; Thurn-Albrecht, T. Nanostructure and Rheology of Hydrogen-Bonding Telechelic Polymers in the Melt: From Micellar Liquids and Solids to Supramolecular Gels. Macromolecules 2014, 47, 2122-2130.

(b) Herbst, F.; Schroeter, K.; Gunkel, I.; Groeger, S.; Thurn-Albrecht, T.; Balbach, J.; Binder, W. H. Aggregation and Chain Dynamics in Supramolecular Polymers by Dynamic Rheology: Cluster Formation and Self-Aggregation. Macromolecules 2010, 43, 10006-10016.

(c) Cortese, J.; Soulié-Ziakovic, C.; Tencé-Girault, S.; Leibler, L. Suppression of Mesoscopic Order by Complementary Interactions in Supramolecular Polymers. J. Am. Chem. Soc. 2012, 134, 3671-3674.

(d) Cortese, J.; Soulié-Ziakovic, C.; Cloitre, M.; Tencé-Girault, S.; Leibler, L. Order-Disorder Transition in Supramolecular Polymers. J. Am. Chem. Soc. 2011, 133, 19672-19675.

${ }^{7}$ Krutyeva, M.; Brás, A. R.; Antonius, W.; Hövelmann, C. H.; Poulos, A. S.; Allgaier, J.; Raduescu, A.; Lindner, P.; Pyckhout-Hintzen W.; Wischnewski A.; Richter D. Association Behavior, Diffusion, and Viscosity of End-Functionalized Supramolecular Poly(ethylene glycol) in the Melt State. Macromolecules 2015, 48, 8933-8946.

${ }^{8}$ Lee, J. S.; Foster, M. D.; Wu, D.T. Effects of Branch Points and Chain Ends on the Thermodynamic Interaction Parameter in Binary Blends of Regularly Branched and Linear Polymers. Macromolecules 2006, 39, 5113-5121.

${ }^{9}$ Allgaier, J. ; Hövelmann, C.H.; Wei, Z.; Staropoli, M.; Pyckhout-Hintzen, W.; Lühmann, N.; Willbold, S. Synthesis and rheological behavior of poly(1,2-butylene oxide) based supramolecular architectures. RSC Adv. 2016, 6, 6093-6106.

${ }^{10}$ Gerstl, C.; Schneider, G. J.; Pyckhout-Hintzen, W.; Allgaier, J.; Willbold, S.; Hofmann, D.; Disko, U.; Frielinghaus, H.; Richter, D. Chain Conformation of Poly(alkylene oxide)s Studied by Small-Angle Neutron Scattering. Macromolecules 2011, 44, 6077-6084.

${ }^{11}$ Allgaier, J.; Willbold, S.; Chang, T. Synthesis of Hydrophobic Poly(alkylene oxide)s and Amphiphilic Poly(alkylene oxide) Block Copolymers. Macromolecules 2007, 40, 518-525.

${ }^{12}$ (a) Radulescu, A.; Szekely, N. K.; Appavou, M. S. KWS-2: Small angle scattering diffractometer. Journal of large-scale research facilities, 1, A29 2015 (b) Radulescu, A.; Pipich, V.; Frielinghaus, H.; Appavou, M. S. KWS-2, the high intensity / wide Q-range small-angle neutron diffractometer for soft-matter and biology at FRM II. Journal of Physics: Conference Series, 2012, 351(1), 012026

${ }^{13}$ (a) Higgins, J. S.; Benoit, H. Polymers and Neutron Scattering, Clarendon: Oxford U.K. 1994. (b) Hammouda, B. Random phase approximation for compressible polymer blends. Journal of NonCrystalline Solids (1994), 172-174, 927-931 
${ }^{14}$ (a) Read, D. J. Mean Field Theory for Phase Separation during Polycondensation Reactions and Calculation of Structure Factors for Copolymers of Arbitrary Architecture. Macromolecules 1998, 31, 899-911. (b) Benoit, H.; Hadziiannou, G. Scattering theory and properties of block copolymers with various architectures in the homogeneous bulk state. Macromolecules 1988, 21, 1449-1464.

${ }^{15}$ (a) Sakurai, S.; Hasegawa, H.; Hashimoto, T.; Hargis, G.; Aggarwal, S. L.; Han, C. C. Microstructure and isotopic labeling effects on the miscibility of polybutadiene blends studied by the small-angle neutron scattering technique. Macromolecules 1990, 23, 451-459.

(b) Bates, F. S.; Wignall, G. D.; Koehler, W. C. Microstructure and isotopic labeling effects on the miscibility of polybutadiene blends studied by the small-angle neutron scattering technique. Phys. Rev. Lett. 1985, 55, 2425-2428.

${ }^{16}$ Greenberg, C. C.; Foster, M. D.; Turner, C. M.; Corona-Galvan, S.; Cloutet, E.; Quirk, R. P.; Butler, P. D.; Hawker, C. Effective Interaction Parameter between Branched and Linear Polystyrene. J. Polym. Sci., Part B: Polym. Phys. 2001, 39, 2549-2561.

${ }^{17}$ Gerstl, C.; Schneider, G. J.; Pyckhout-Hintzen, W.; Allgaier, J.; Richter, D.; Alegria, A.; Colmenero, J. Segmental and Normal Mode Relaxation of Poly(alkylene oxide)s Studied by Dielectric Spectroscopy and Rheology. Macromolecules 2010, 43, 4968-4977.

${ }^{18}$ Zamponi, M.; Wischnewski, A; Monkenbusch, M.; Willner, L.; Richter, D.; Likhtman, A. E.; Kali, G.; Farago, B. Molecular Observation of Constraint Release in Polymer Melts. Phys. Rev. Lett. 2006, 96, 238302.

${ }^{19}$ (a) Doi, M.; Graessley, W. W.; Helfand, E.; Pearson, D. S. Dynamics of Polymers in Polydisperse Melts. Macromolecules 1987, 20, 1900-1906.

(b) Watanabe, H. Viscoelasticity and dynamics of entangled polymers. Prog. Polym. Sci. 24 (1999) 12531403

${ }^{20}$ (a) van Ruymbeke, E.; Vlassopoulos, D.; Mierzwa, M.; Pakula, T.; Charalabidis, D.; Pitsikalis, M.; Hadjichristidis, N. Rheology and Structure of Entangled Telechelic Linear and Star Polyisoprene Melts. Macromolecules 2010, 43, 4401-4411.

(b) Stadler, F. J.; Pyckhout-Hintzen, W.; Schumers, J. M.; Fustin, C. A.; Gohy, J. F.; Bailly, C. Linear Viscoelastic Rheology of Moderately Entangled Telechelic Polybutadiene Temporary Networks. Macromolecules 2009, 42, 6181-6192.

${ }^{21}$ (a) Inkson, N. J.; Graham, R. S.; McLeish, T. C. B.; Groves, D. J.; Fernyhough, C. M. Viscoelasticity of Monodisperse Comb Polymer Melts. Macromolecules 2006, 39, 4217-4227.

(b) Daniels, D. R.; McLeish, T. C. B.; Crosby, B. J.; Young, R. N.; Fernyhough, C. M. Molecular Rheology of Comb Polymer Melts. 1. Linear Viscoelastic Response. Macromolecules 2001, 34, 7025-7033.

(c) Kapnistos, M.; Vlassopoulos, D.; Roovers, J.; Leal, L. G. Linear Rheology of Architecturally Complex Macromolecules: Comb Polymers with Linear Backbones. Macromolecules 2005, 38, 7852-7862. 
${ }^{22}$ (a) Baumgaertel, M.; Schausberger, A.; Winter, H. H. The relaxation of polymers with linear flexible chains of uniform length. Rheologica Acta 1990, 29, 400.

(b) Baumgaertel, M.; De Rosa, M. E.; Machado, J.; Masse, M.; Winter, H. H. The relaxation time spectrum of nearly monodisperse polybutadiene melts. Rheologica Acta 1992, 31, 75-82.

${ }^{23}$ Monkenbusch, M. et al. (submitted to Physical Review Letters - April 2016)

${ }^{24}$ Stadler, F. J.; Bailly, C. A new method for the calculation of continuous relaxation spectra from dynamic-mechanical data. Rheologica Acta 2009, 48, (1), 33-49.

${ }^{25}$ McDougall, I.; Orbey, N. J.; Dealy, M. Inferring meaningful relaxation spectra from experimental data. J. Rheol. 2014, 58, (3), 779-797. 


\section{For Table of Contents Use Only}

Hydrogen Bonding in a Reversible Comb Polymer Architecture: A Microscopic and Macroscopic Investigation.

M. Staropoli, A. Raba, C. Hövelmann, M. Krutyeva, J. Allgaier, M.S. Appavou, U. Keiderling, F.J. Stadler, W.Pyckhout-Hintzen, A. Wischnewski, D. Richter

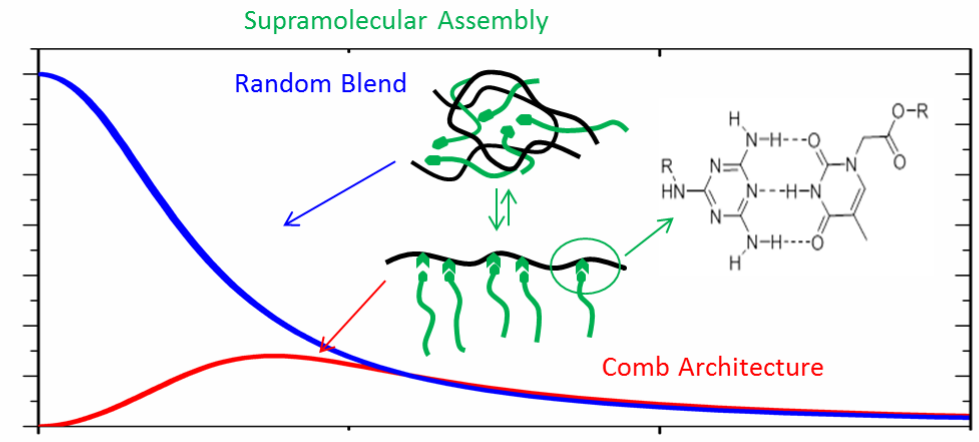

Table of Contents Graphic 